\title{
Trabajo interdisciplinar en tecnificación en deportes de invierno: un abordaje desde la psicología del deporte
}

\author{
Alba Villamediana Sáez y Paula Lázaro de la Iglesia \\ Federación Madrileña de Deportes de Invierno, España
}

RESUMEN: El área de psicología de la Federación Madrileña de Deportes de Invierno organizó 5 jornadas de tecnificación: esquí alpino, mushing, 2 de patinaje sobre hielo y 1 de hockey hielo. En un total de 15 sesiones entre 1-2 horas de duración se llevó a cabo una planificación coordinada con los entrenadores y vocales de cada deporte, con el fin de favorecer un trabajo integrado que tuviera aplicabilidad en sus entrenamientos durante las jornadas. Se pudo ver que esta forma de trabajo conjunto favorece la comunicación del equipo técnico interdisciplinar que tendrá como consecuencia una formación más completa del deportista. Además, el entrenamiento en habilidades psicológicas (como la orientación motivacional y la gestión de pensamientos) forma un conjunto coherente dentro del entrenamiento y junto al contexto de reflexión, autoconocimiento y debate establecido durante las sesiones, permitió ampliar las formas de enfrentarse a su deporte, tanto en entrenamientos como en competiciones.

PALABRAS CLAVES: Psicología del deporte, trabajo interdisciplinar, deportes de invierno, tecnificación, psicólogo del deporte.

\section{Interdisciplinary work in technology in winter sports: an approach from the sport psychology}

ABSTRACT: The psychology department of the Madrid Winter Sports Federation organized 5 high performance group sessions: one session each of alpine skiing, mushing, and ice hockey, and two of figure skating. A total of 15 sessions lasting 1-2 hours were held to coordinate planning with the coaches and spokepersons of each sport modality in order to promote an integrated work that could be applied during training. These sessions showed that such a joint effort promotes communication of the interdisciplinary technical team, which will help athletes achieve a more complete training. In addition, training in psychological skills (such as goal setting and thought management) is part of a coherent training. The context of reflection, self-knowledge, debate and practice established during the sessions allowed athletes to broaden the ways of dealing with their sport both during training and in competitions.

KEYWORDS: Sport psychology, interdisciplinary work, winter sports, technification, sport psychologist.

Trabalho interciplinar em tecnicização em desportos de inverno: uma abordagem da psicologia do desporto

RESUMO: Na área de psicologia da Federação de Desportos de Inverno de Madrid, foram organizados 5 dias de tecnicização: esqui alpino, mushing, 2 dias de patinagem no gelo e 1 dia de hóquei no gelo. Num total de 15 sessões com a duração de 1 a 2 horas, foi realizado um planeamento coordenado com os treinadores e os porta-vozes de cada desporto, a fim de promover um trabalho integrado que tivesse aplicabilidade dos seus treinos, durante as sessões. Pudemos observar que essa forma de trabalho conjunto favorece a comunicação da equipa técnica interdisciplinar, o que resultará numa formação mais completa do atleta. Além disso, o treino de capacidades psicológicas (como a orientação motivacional e a gestão de pensamentos) forma um conjunto coerente no âmbito do

\footnotetext{
Alba Villamediana Sáez es Graduada en Psicología (UCM). Máster en psicología de la actividad física y el deporte (UAM). Experta en coaching deportivo (COPM).

Paula Lázaro de la Iglesia es Graduada en Psicología (USAL). Máster en psicología de la actividad física y el deporte (UAM).

La correspondencia sobre este artículo debe enviar a las autoras al Área de psicología de la Federación Madrileña de Deportes de Invierno. Avenida Salas de los infantes, 1. Planta 5. Edificio el Barco. Email:
} 
treino e, juntamente com o contexto de reflexão, autoconhecimento e debate estabelecido durante as sessões, permitiu expandir as formas de lidar com o seu desporto, tanto nos treinos como nas competições.

PALAVRAS-CHAVE: psicologia do desporto, trabalho interdisciplinar, desportos de inverno, tecnicização, psicólogo do desporto.

Artículo recibido: 14/03/2020 | Artículo aceptado: 31/05/2020

Los deportes de hielo y de nieve tienen una amplia tradición a nivel europeo a pesar de tratarse de deportes minoritarios cuando nos referimos a la competición. A nivel nacional, se agrupan en federaciones diferenciadas (Real Federación Española de Deportes de Invierno y Real Federación Española de Deportes de Hielo), pero a nivel autonómico, cada comunidad engloba las disciplinas de hielo y las de nieve en una misma institución. En el caso de Madrid hablamos de la Federación Madrileña de Deportes de Invierno (FMDI, n.d.).

Dentro de los deportes de nieve más conocidos está esquí alpino, con más de 100 años de historia, cuya competición a nivel olímpico empieza en los Juegos de 1936 de Garmisch-Partenkirchen, Alemania (Real Federación Española de Deportes de Invierno [RFEDI], n. d.).

El mushing es otro deporte dentro de la modalidad de nieve que agrupa la Federación Madrileña de Deportes de Invierno (FMDI). Tal y como lo definía Pares en su libro (1999), el mushing es un deporte que consiste en desplazarse corriendo o con un vehículo de ruedas o esquís tirado por un número variable de perros.

En la modalidad de hielo nos encontramos con el patinaje artístico sobre hielo, abarcando diferentes disciplinas: individual, parejas, danza, sincronizado... Es deporte olímpico desde la primera edición de los juegos de invierno, en Chamonix, pero antes de ello fue parte del programa de los juegos de verano de Londres de 1908 (Federación Española de Deportes de Hielo [FEDH], n. d.).

El hockey, como deporte colectivo, nació en la ciudad de Quebec, en Montreal. Es actualmente jugado en aproximadamente 68 países de forma oficial y cuya presencia olímpica data de los años 20 (FEDH, n. d.).

Las diferentes disciplinas deportivas están representadas en la federación autonómica por los numerosos clubes y deportistas de la comunidad. De esos deportistas surge una selección anual con aquellos cuyos resultados han sido destacados. Una de las iniciativas de la FMDI es realizar actividades formativas para estos deportistas seleccionados que forman los equipos madrileños. Estas jornadas de tecnificación se organizan como concentraciones interdisciplinares que tienen como objetivo, desde la iniciación a la competición, una formación completa del deportista (FMDI, n. d.).

El área de psicología de la FMDI fue creada en la temporada 2015-2016 por Alba Villamediana Sáez. Tras dos años de trabajo con deportistas a nivel individual y colectivo en los clubes federados se incluyó al equipo Paula Lázaro de la Iglesia quien continuó dicho trabajo, reforzando la implementación del entrenamiento mental en los deportistas y clubes de las disciplinas de hielo y nieve.

Las jornadas de tecnificación ya se realizaban antes de la incorporación del Área de Psicología del Deporte como parte de la estructura deportiva de la FMDI. Es después de su creación, cuando empezamos a planificar estas jornadas de una manera interdisciplinar, intentando que todo el contenido formativo propuesto tuviera consonancia e incluyendo la preparación psicológica de forma específica para cada disciplina. En las etapas de iniciación y deporte base es precisamente dónde pueden potenciarse todas las habilidades psicológicas que en un futuro pueden tener un valor clave en el rendimiento deportivo adulto (Marsillas et al., 2014). Por esa razón, el rol del psicólogo cobra especial relevancia durante la formación de los deportistas (Pérez y Llames, 2010).

Un enfoque interdisciplinar de la psicología del deporte puede definirse como "un proceso de evaluación y administración en donde profesionales de la salud comparten su conocimiento, aptitudes, y destrezas, según se necesiten, para la aplicación o práctica interdisciplinaria del cuidado de la salud desde al menos dos profesiones distintas" (Moreau y Nabhan, 2012, p.346). Por esa razón, Cohen (2004) afirma que una de las principales funciones de un trabajo interdisciplinar integrado se basa en la intercomunicación efectiva y una planificación integrada que abarque todas las necesidades de los deportistas y del rendimiento deportivo.

El trabajo interdisciplinar realizado en las jornadas de tecnificación anuales, desde la incorporación del área de psicología, está basado en la labor que llevan realizando durante la última década en el Centro de Excelencia de la Asociación de Snowboard y de Esquí Estadounidense en Park City, Utah (Moreau y Nabhan, 2012). Este centro cuenta con un área educacional, varias zonas de entrenamiento y servicios médicos que pueden usar tanto deportistas, técnicos, padres y madres... 
En las edades que se encuentran los deportistas de las jornadas, los entrenadores se convierten en una de las figuras principales y modelo para los deportistas. Es por ello por lo que resulta imprescindible contar con un trabajo colaborativo de éstos, sumando a su experiencia, conocimiento y profesionalidad, la aportación de otras áreas como puede ser la psicología (Pérez y Llames, 2010). Por esa razón, una de las principales funciones de un trabajo interdisciplinar integrado se basa en la intercomunicación efectiva y una planificación integrada que abarque todas las necesidades de los deportistas y del rendimiento deportivo. Una colaboración exitosa requiere de un ambiente de respeto y confianza compartidos, con un establecimiento de relaciones fortalecidas (Moreau y Nabhan, 2012).

Estas afirmaciones sobre los beneficios del trabajo interdisciplinar no provienen solo de la Psicología del Deporte sino también de las otras Ciencias del Deporte, por lo que el trabajo expuesto se centra en incidir en la importancia de la integración de los conocimientos de los profesionales que trabajan en el ámbito deportivo. Con este fin vamos a presentar el trabajo psicológico realizado en las 5 jornadas diferentes de tecnificación, distribuidas en un total de 15 sesiones.

\section{Experiencia profesional}

Las necesidades psicológicas que nos encontramos en los deportistas de invierno con los que trabajamos en las jornadas de tecnificación (esquí alpino, mushing, patinaje sobre hielo y hockey hielo) son variadas y diferentes para cada disciplina, aunque sí tienen puntos en común que están relacionados con su edad, nivel deportivo adquirido y características de la competición. Los trabajos relacionados con entrenamiento psicológico en edades jóvenes y tecnificación están orientados en la necesidad de trabajar desde un punto de vista preventivo para evitar una orientación excesiva al resultado (Pérez y Llames, 2010). Por esa razón, dentro de las jornadas de tecnificación en las que trabajamos tuvimos dicho objetivo como orientación general común.

Al inicio de la temporada realizamos una evaluación de las necesidades psicológicas de cada deporte y fueron los propios directores técnicos de la FMDI quien, concienciados de la importancia de los aspectos psicológicos en su deporte, decidieron contar con dicha formación dentro de las jornadas de tecnificación que organizaban cada año, donde se congregaban los deportistas seleccionados de cada club madrileño de las diferentes disciplinas.

El trabajo se realizó en la temporada 2017-2018 con un total de 5 jornadas de tecnificación distribuidas en 15 sesio- nes de entrenamiento mental destinadas a los deportistas seleccionados de los equipos madrileños. Coordinamos la planificación del trabajo psicológico con las actividades físicas, técnicas y tácticas planteadas por los entrenadores de los respectivos deportes. Dentro de una metodología practica y lúdica en dinámicas colectivas, adaptamos el contenido de forma específica a los deportistas asistentes.

En el grupo de esquí alpino participaron 9 deportistas (6 chicos y 3 chicas) con edades comprendidas entre los 15 y 17 años pertenecientes a cuatro clubes asociados a la federación. Por su parte, el grupo de patinaje se componía, en la primera concentración, de 12 chicas y 2 chicos entre los 13 y 16 años y en la segunda concentración de patinaje, el número de patinadores se duplicó, teniendo un total de 33 divididos en 26 chicas y 7 chicos. En el caso de mushing, el grupo que realizó la concentración estaba compuesto por 7 deportistas, 4 mujeres y 3 hombres pertenecientes a la categoría absoluta. Y, por último, la concentración de hockey sobre hielo estaba dirigida a las categorías sub14 y sub17 por lo que contábamos con 37 deportistas: 30 jugadores y 7 jugadoras.

Todos los deportistas mencionados realizaban su preparación física y técnica semanal en sus respectivos clubes, y acudían a dichas tecnificaciones con el fin de realizar una formación completa e integrada, sirviendo también de seguimiento de los deportistas por parte de la federación. Todos compatibilizaban su práctica deportiva con sus trabajos y estudios obligatorios.

En relación a la experiencia con la Psicología del Deporte de los deportistas cabe destacar que algunos de ellos ya habían tenido contacto a través de sesiones individuales con las psicólogas del área, mientras que otros no contaban con experiencia de ningún tipo y se trataba de su primer acercamiento.

\section{Organización del trabajo interdisciplinar}

Durante el transcurso de las jornadas de tecnificación de cada disciplina tuvimos una comunicación directa y constante con los entrenadores y con los responsables de las concentraciones. La premisa era que todo el trabajo se realizara en la misma dirección, por lo que necesitábamos saber qué temáticas iban a trabajar el resto de los profesionales para incluir una programación de herramientas que fueran en la misma línea. Un ejemplo de esta coordinación interdisciplinar y comunicación constante lo encontramos en el grupo de tecnificación de patinaje en el que, junto con la directora técnica de la FMDI y la vocal de patinaje, diseñamos unos objetivos psicológicos alrededor de la orientación mo- 
tivacional a la tarea y mejora de la capacidad de atención y pensamientos, de forma que tuvieran consonancia con los objetivos que querían alcanzar dentro del hielo (trabajo técnico de filos y desplazamientos). Esto se llevó a cabo con una reunión previa a las jornadas y otra de evaluación post-jornada.

Con este ejemplo queda patente que la comunicación con el resto de los miembros del equipo interdisciplinar resulta esencial para que haya un mayor éxito y una optimización del rendimiento, evitando que se contradigan o solapen las funciones (Moreau y Nabhan, 2012). Y así, además, poder elaborar una metodología fuera del hielo o de la nieve que tuviera transferencia dentro, teniendo en cuenta las necesidades y demandas de los deportistas para que la aplicación de las herramientas sea posible (García-Mas y Gimeno, 2008).

La distribución entre el número de sesiones de psicología y organización con el resto de las actividades fue diferente en las distintas disciplinas, tal y como se detalla en los apartados siguientes.

\section{Trabajo psicológico en las jornadas de tecnificación}

Como evaluación previa se aplicó el Cuestionario Psicológico de Rendimiento deportivo (Gimeno et al., 2001). Con ello pudimos establecer un perfil de los deportistas y una detección inicial de necesidades del grupo con el que posteriormente se trabajaría. También hablamos con los entrenadores y encargados de los comités, para detectar las necesidades y planificación de los contenidos de las jornadas.

El trabajo psicológico con los deportistas se dedicó al entrenamiento de las habilidades psicológicas básicas (Dosil, 2008), principalmente al establecimiento de objetivos, manejo de pensamientos y atención.

La organización de las sesiones estaba dividida en 4 fases: una primera parte de seguimiento general de la semana; una breve introducción teórica del contenido a tratar, aplicación práctica a través de una actividad lúdica; y finalmente, hicimos una reflexión conjunta sobre la forma de aplicar lo aprendido (Villamediana, 2018).

\section{Trabajo psicológico realizado con esquí alpino}

El trabajo psicológico en las jornadas de tecnificación de esquí alpino se realizó en la Residencia de Navacerrada, en el Puerto de Navacerrada, en el periodo de pretemporada previo a la apertura de las estaciones. En dichas jornadas los deportistas realizaron marchas por la montaña, un programa de preparación física adaptada al esquí y una hora de sesión de psicología aplicada al deporte que sirvió de cierre de un día de formación integral.

La sesión duró 1 hora y la realizamos de forma grupal en una sala habilitada para ello (temperatura, iluminación, sillas y espacio abierto para las actividades), facilitando así las actividades junto con el debate y la reflexión planificadas.

La sesión se basó en establecer los contenidos básicos de una correcta orientación motivacional hacia la tarea (Gavino, 2008) a través de la técnica de establecimiento de objetivos (Cox, 2008; Dosil, 2008) e introducción a la gestión de pensamientos (Carrasco y Espinar, 2008).

Tras una reflexión conjunta y refuerzos grupales pudimos realizar un debate acerca de los pensamientos generados en situaciones típicas en su deporte en el camino hacia sus objetivos. Los deportistas pudieron compartir pensamientos en relación a preocupaciones, miedos, formas de animarse y debatir con ello la utilidad de dichos pensamientos y en caso negativo, los posibles pensamientos alternativos que podrían servir de utilidad en el camino a los objetivos planteados.

\section{Trabajo psicológico realizado con mushing}

La concentración del equipo de mushing se realizó también en la residencia de Navacerrada, en el Puerto de Navacerrada. Se trata de una jornada de concentración de un solo día en la que los deportistas tenían una sesión de entrenamiento acompañados de sus perros, posteriormente una sesión de 2 horas de psicología deportiva y para finalizar, otra sesión de la misma duración de preparación física específica.

Para la sesión de psicología deportiva contamos con una sala amplia dentro de la residencia que permitió llevar a cabo de forma cómoda tanto las actividades prácticas como las reflexiones conjuntas.

El contenido que decidimos abordar dentro de nuestra planificación psicológica estaba respaldado por el momento de pretemporada previa al Campeonato de Europa en el que se encontraban los deportistas.

El trabajo se centró en llevar a cabo un análisis de la temporada anterior para que los mushers pudieran reflexionar sobre los objetivos cumplidos, dificultades, soluciones intentadas, sensaciones y rendimiento de su temporada previa, considerando que para planificar una buena temporada se debe analizar las necesidades y demandas que te han surgido en la anterior. Para que la actividad fuera más práctica, se utilizó la metáfora de "la brújula" en la que los deportistas tenían que dibujar una brújula y en su interior escribir toda esa información que consideraban relevante para se- 
guir avanzando. De esta manera, una vez llevado a cabo el análisis, pudimos trazar la línea de trabajo y de objetivos que querían seguir.

\section{Trabajo psicológico realizado con patinaje sobre hielo}

En esta disciplina se pudo contar con dos jornadas anuales: una al inicio de la temporada y otra al final. Se realizó en la pista de hielo La Nevera de Majadahonda, pudiendo contar con una sala diáfana y amplia perfecta para el desempeño de las actividades prácticas.

En septiembre de 2017, al inicio de la temporada, se realizaron 4 días de concentración en el que los y las patinadoras participaban en entrenamientos en hielo, entrenamientos físicos fuera del hielo, clases de expresión corporal y sesiones de psicología del deporte.

En este último, el contenido se basó en realizar una introducción básica a los contenidos de la psicología del deporte, junto con el fomento de una orientación a la tarea como principal factor de prevención de futuros síntomas de ansiedad precompetitiva, presión y retirada temprana (García-Mas y Gimeno, 2008).

Además del trabajo mencionado, pudimos realizar dos sesiones aplicadas, basadas en la preparación psicológica de la competición y una sesión final de relajación.

A través de una dinámica lúdico-práctica expusimos viñetas con situaciones típicas de competición y entre todos los patinadores y patinadoras establecieron conductas que mejoraban su rendimiento a nivel de concentración y gestión de la ansiedad (Marsillas, et al., 2014).

En la última jornada realizamos una sesión completa de relajación muscular progresiva. Tras una primera fase de explicación llevamos a cabo un ejercicio de 25 minutos de relajación muscular progresiva de 16 grupos musculares (Soto, 2019).

La segunda concentración de patinaje se realizó en junio de 2018, a finales de temporada y volvió a desarrollarse durante 4 días, aunque esta vez se trataba de dos grupos de patinadores diferenciados por edad o nivel de competición, desarrollando así un total de 8 sesiones (2 sesiones de psicología al día, una con cada grupo).

Durante la primera jornada, nos centramos en realizar un análisis de la temporada y posterior establecimiento de objetivos, no tanto con la meta de fijar objetivos para la temporada siguiente sino para concienciar a los deportistas que, si se ponían objetivos para las jornadas de tecnificación, tenían más probabilidades de optimizar su rendimiento (Dosil, 2008). Las dos sesiones siguientes nos centramos en la gestión de pensamientos y emociones de los deportistas, siguiendo las bases de la terapia emotiva y el modelo A-B-C de Albert Ellis (1955, citado en Toro, 2014). Realizamos también de forma complementaria una serie de actividades de expresión emocional, muy relacionada con el componente artístico y de expresión de su deporte.

Por último, cerramos la tecnificación con una sesión de pautas más aplicadas sobre el foco atencional, los diferentes tipos de atención y su relevancia dentro del rendimiento deportivo (Aguirre-Lozaia et al., 2015).

\section{Trabajo psicológico realizado con Hockey hielo}

La tecnificación de hockey hielo se realizó también en la pista de hielo de La Nevera en Majadahonda durante 3 días en los cuales los deportistas contaban con preparación técnica y táctica dentro del hielo, entrenamiento en seco, una sesión táctica de video y una sesión de psicología deportiva.

La sesión de psicología deportiva se llevó a cabo uno de los días de la tecnificación en el espacio de 1 hora con un único grupo que contaba con 37 jugadores de las categorías sub14 y sub17.

Al ser un deporte de equipo y sin experiencia en psicología del deporte, el primer objetivo que fijamos de forma conjunta con el director técnico de hockey de la federación era realizar una introducción a la implicación del componente mental y relacional dentro del hockey. Por otro lado, estando en consonancia con el cuerpo técnico, centramos el trabajo en los diferentes procesos de equipo y cómo estos pueden afectar al desempeño y rendimiento del grupo. Siendo el punto central del contenido la cohesión, un constructo que hace referencia a la tendencia relacional entre los miembros de un grupo de cara a conseguir objetivos (Bowers et al., 2017).

Otro de los objetivos principales era realizar la sesión de manera más lúdica al tratarse de un grupo grande y contar únicamente con 1 hora. Aun así, comenzamos la sesión definiendo qué es la psicología deportiva y para qué puede servir y posteriormente dividimos el grupo en pequeños subgrupos para darles la tarea de destacar qué valores consideraban importantes dentro de un buen equipo. Utilizamos dinámicas para trabajar la comunicación, un constructo muy relevante dentro de la cohesión de equipo en los deportes colectivos (Bohórquez y García-Mas, 2014; Dosil, 2008) y del juego del hockey sobre hielo de manera específica al tratarse de un deporte en el que la velocidad del juego es tan alta que cuanta más comunicación, confianza y cohesión tengas con tus compañeros de equipo, mejor será el rendimiento (Tutte et al., 2020).

En la Tabla 1 se presenta un resumen de las actividades organizadas para cada deporte. 
Tabla 1. Objetivos y técnicas psicológicas empleadas en las diferentes jornadas de tecnificación interdisciplinar en la FMDI

\begin{tabular}{|c|c|c|c|}
\hline Deporte & $\begin{array}{l}\text { Número de } \\
\text { sesiones }\end{array}$ & Objetivos de las jornadas & Técnicas psicológicas empleadas \\
\hline ESQUÍ ALPINO & $\begin{array}{l}1 \text { sesión de } \\
1 \text { hora }\end{array}$ & $\begin{array}{l}\text { Introducción a la psicología del deporte. } \\
\text { Orientación motivacional a la tarea. } \\
\text { Mejorar capacidad de detección de pensamientos. }\end{array}$ & $\begin{array}{l}\text { Establecimiento de objetivos de tarea (Gavino, 2008). } \\
\text { Terapia cognitiva y detención de pensamientos } \\
\text { (Carrasco y Espinar, 2008). }\end{array}$ \\
\hline MUSHING & $\begin{array}{l}1 \text { sesión de } \\
2 \text { horas }\end{array}$ & $\begin{array}{l}\text { Introducción a la psicología del deporte. } \\
\text { Análisis y evaluación del feedback de la temporada } \\
\text { anterior. } \\
\text { Búsqueda de soluciones y reconocimiento de los } \\
\text { logros en el rendimiento. } \\
\text { Establecimiento de objetivos y orientación hacia la } \\
\text { tarea. }\end{array}$ & $\begin{array}{l}\text { Establecimiento de objetivos de tarea (Gavino, 2008). } \\
\text { Análisis de la información. } \\
\text { Terapia Breve Centrada en Soluciones (Beyebach y } \\
\text { Herrero de la Vega, 2010). }\end{array}$ \\
\hline $\begin{array}{l}\text { PATINAJE SOBRE } \\
\text { HIELO (Inicio de } \\
\text { temporada) }\end{array}$ & $\begin{array}{l}4 \text { sesiones } \\
\text { de } 1 \text { hora }\end{array}$ & $\begin{array}{l}\text { Introducción a la psicología del deporte } \\
\text { Orientación motivacional a la tarea. } \\
\text { Mejorar capacidad de detección de pensamientos. } \\
\text { Detectar variables intervinientes y sus consecuentes } \\
\text { en el rendimiento en competición. } \\
\text { Aplicación de estrategias de relajación en competición. }\end{array}$ & $\begin{array}{l}\text { Establecimiento de objetivos de tarea (Gavino, 2008). } \\
\text { Terapia cognitiva y detención de pensamientos } \\
\text { (Carrasco y Espinar, 2008). } \\
\text { Rutinas precompetitivas (Cox, 2008; Dosil, 2008). } \\
\text { Relajación progresiva de Jacobson (Soto, 2019). }\end{array}$ \\
\hline $\begin{array}{l}\text { PATINAJE SOBRE } \\
\text { HIELO (Final de } \\
\text { temporada) }\end{array}$ & $\begin{array}{l}8 \text { sesiones } \\
\text { de } 1 \text { hora }\end{array}$ & $\begin{array}{l}\text { Introducción a la psicología del deporte. } \\
\text { Establecimiento de objetivos de tarea y temporalizados } \\
\text { para la tecnificación. } \\
\text { Gestión de pensamientos y su implicación en las } \\
\text { emociones } \\
\text { Dónde prestamos atención en cada momento y cómo } \\
\text { podemos dirigirla. }\end{array}$ & $\begin{array}{l}\text { Establecimiento de objetivos de tarea (Gavino, 2008). } \\
\text { Terapia cognitiva y detención de pensamientos } \\
\text { (Carrasco y Espinar, 2008). } \\
\text { Modelo A-B-C de Albert Ellis (1955, citado en Toro, } \\
\text { 2014). } \\
\text { Estilos atencionales: interno /externo; amplio/estre- } \\
\text { cho (Aguirre-Lozaia, et al., 2015). } \\
\text { Técnicas de cambio del foco atencional (Aguirre-Lo- } \\
\text { zoia, et al., 2015). }\end{array}$ \\
\hline HOCKEY HIELO & $\begin{array}{l}1 \text { sesión de } \\
1 \text { hora }\end{array}$ & $\begin{array}{l}\text { Introducción a la psicología del deporte } \\
\text { Valores deportivos en el hockey sobre hielo } \\
\text { Procesos de equipo en hockey sobre hielo } \\
\text { Cómo influye la comunicación en la consecución de un } \\
\text { objetivo }\end{array}$ & $\begin{array}{l}8 \text { claves para construir un equipo exitoso (Bohórquez } \\
\text { y García - Mas, 2014) }\end{array}$ \\
\hline
\end{tabular}

\section{Evaluación de programas e intervenciones}

La evaluación final se realizó únicamente a través de encuestas de satisfacción subjetivas, con un cuestionario de preguntas abiertas realizadas únicamente a los deportistas con el fin de favorecer la libre expresión, junto con la observación de lo aprendido en su día a día de entrenamientos y competiciones. Además, la comunicación directa con los entrenadores permitió comprobar la aplicabilidad de los aprendizajes durante los entrenamientos y sirvió para establecer de manera conjunta nuevas líneas de mejora para futuros trabajos conjuntos.

En la reunión final con los entrenadores quedó patente la satisfacción del trabajo en equipo. Bajo la ob- servación de los técnicos, se expuso el éxito de la metodología conjunta de cara al rendimiento de los y las deportistas en la aplicación de técnicas psicológicas en los entrenamientos.

Por otro lado, aunque no podemos establecer una relación directa entre el rendimiento, la aportación de herramientas psicológicas y el resultado, sí podemos afirmar que 6 de los deportistas que participaron en las jornadas de tecnificación, posteriormente han demandado un trabajo individual ( 1 de esquí alpino, 1 de hockey hielo y 4 de patinaje). Además, un club de patinaje sobre hielo solicitó el trabajo específico con sus patinadores, entrenadores y madres/padres del club. 


\section{DISCUSIÓN}

Con la inclusión de la preparación psicológica en las jornadas de tecnificación buscamos fomentar la orientación motivacional hacia la tarea, utilizando una planificación de metas que sirva para tener siempre un plan de acción y así facilitar la concentración en el rendimiento y la evaluación de los progresos (Pérez y Llames, 2010). Por otro lado, el objetivo común del planteamiento de las tecnificaciones se trataba de promover el trabajo interdisciplinar para conseguir un desarrollo más completo de los deportistas y una mayor optimización de su rendimiento.

Para que el trabajo psicológico forme parte de la preparación de los deportistas y se normalice dentro de su rutina, es importante asociar las actividades prácticas, el debate y la reflexión al contenido del entrenamiento, las características de la disciplina deportiva y los aspectos psicológicos más implicados. Es por eso que establecimos una comunicación eficaz y fluida con los directores técnicos de cada tecnificación, así como con los responsables de las diferentes áreas de trabajo. Así, también, acabamos con la tradicional imagen del psicólogo caracterizado por ser un trabajo basado en la relación exclusiva con el deportista y poco integrado con las otras áreas del entrenamiento. (Gordillo et al., 2011). Además, en este sentido, desde el planteamiento interdisciplinar, es como se llegó al trabajo de los objetivos psicológicos planteados, teniendo en cuenta que la meta fundamental del trabajo era favorecer un trabajo integrado que tuviera aplicabilidad en sus entrenamientos durante las jornadas. Además, en una concentración de tecnificación el foco se centra en el aprendizaje y la adaptación de los deportistas a los contenidos de entrenamiento, presentes y futuros, incluyendo herramientas que normalmente no se les presentan en su entrenamiento cotidiano (Pradas y Reverter, 2011).

Por lo tanto, se puede extraer del presente trabajo, que además de los conocimientos y competencias propias de la psicología, y en concreto de la psicología del deporte, el psicólogo dentro de un equipo interdisciplinar debe contar con: una buena relación profesional con el equipo; habilidades comunicativas y de escucha; capacidad de argumentar y convencer a través de la palabra y la buena práctica profesional; respeto al trabajo y responsabilidades del resto de profesionales al cargo de los deportistas y, por supuesto una buena capacidad de autocrítica y reflexión (Pérez y Llames, 2010).

En definitiva, no es cuestión únicamente de saber trabajar de forma individual con los deportistas, sino de entender que la manera de conseguir una optimización en nuestro trabajo como psicólogos y en el rendimiento del propio deportista, es a través de la colaboración continua con el resto de los profesionales que intervienen en el desarrollo deportivo.

\section{Limitaciones y futuras líneas de trabajo}

Las jornadas de tecnificación interdisciplinar son una gran iniciativa de la Federación Madrileña de Deportes de Invierno que sustenta la propuesta de actividad deportiva para sus deportistas, pensando en su desarrollo completo. Partiendo de esta base, hay aspectos que tendríamos en cuenta para mejorar de cara a un futuro y otros que ya se han planteado para las siguientes temporadas.

Como una de las principales limitaciones y futuras líneas de mejora en cuanto al trabajo psicológico en las jornadas de tecnificación, se propone un trabajo más personalizado con cada deportista, puesto que el trabajo de dinámicas grupales, aunque tiene numerosas ventajas, cuenta con el inconveniente de la difícil adaptación a cada necesidad de cada deportista y modalidad (García-Mas y Vicens, 2003). Además, buscamos que el número de jornadas por temporada aumente y el período de tiempo entre una tecnificación y otra, disminuya, para así acercarnos más al objetivo de realizar un seguimiento real de los deportivas y cubrir sus necesidades.

Es una línea en la que ya se está comenzando a trabajar a medio y largo plazo. Cada disciplina se ha encargado de diseñar un plan de tecnificación específico para su deporte, con unos objetivos anuales que cumplir. Por ejemplo, en el caso de patinaje sobre hielo, se ha planteado de forma concreta que el equipo de tecnificación sea siempre el mismo, incluyendo el Área de Psicología del Deporte, y que las tecnificaciones para los deportistas seleccionados como Equipo de Madrid, se realicen cada 15 días.

Nosotras, como Área de Psicología del Deporte, hemos continuado con el seguimiento de los deportistas, tanto de manera individual o a nivel de club con aquellos que nos lo solicitan como mediante la evaluación inicial de principio de temporada que continuamos realizando. Además, desde las jornadas de tecnificación expuestas anteriormente, hemos continuado formando parte de las siguientes concentraciones. A nivel interdisciplinar, también hemos mantenido la comunicación continua con el equipo de profesionales que trabajan alrededor de los deportistas a lo largo de la temporada. De esta forma, habrá una evolución más progresiva y adaptada de las variables psicológicas entrenadas, poniendo en práctica en entrenamientos y competiciones los contenidos trabajados en las sesiones (Llames, 1994).

Otra de las limitaciones que hoy en día nos seguimos encontrando, reside en el tiempo que tienen los deportistas fuera de sus horarios de entrenamiento de club y competiciones, así como el coste adicional que supone llevar a cabo las jornadas de tecnificación. Por estas razones es importante la presentación e implantación de proyectos anuales, 
para no caer en la rutina del propio deporte y dejar en el olvido el seguimiento real y adaptado a los deportistas.

Además, de cara a un futuro, nuestra intención es formar parte de las jornadas de tecnificación de todas las disciplinas deportivas que acoge la FMDI, no sólo mediante el trabajo con los deportistas sino también cogiendo como foco de actuación a los entrenadores. El objetivo de este planteamiento es que estén formados en los aspectos psicológicos que forman parte del día a día de su disciplina y las necesidades que puedan tener sus deportistas. Un ejemplo de ello es el trabajo que se inició a raíz de las jornadas de tecnificación, con un club de la FMDI en el que se programaron sesiones específicas para los técnicos.

El trabajo, por tanto, está orientado a la formación continua para buscar un futuro máximo rendimiento, sin un límite estricto de tiempo. Por esta razón, tenemos la responsabilidad de fomentar una preparación psicológica integrada y adaptada a las otras tres áreas imprescindibles de la formación deportiva: técnica, táctica y físico, para que así la trasferencia y aplicación a las necesidades del deporte, sea mucho más fácil y eficaz.

\section{REFERENCIAS}

Aguirre-Lozaia, H. H., Ayala, C. F. y Ramos, S. (2015). La atención-concentración en el deporte de rendimiento. Educación Física y Deporte, 34(2), 409-428. https://doi.org/10.17533/udea. efyd.v34n2a06

Beyebach, M. y Herrero de la Vega, M. (2010). 200 tareas en terapia breve. Herder Editorial.

Bohórquez, R. y García-Más, A. (2014). 8 claves para construir equipos exitosos. En M. Roffé y S. Rivera (Coords.), Entrenamiento Mental en el Fútbol Moderno (pp. 390-407). Fútbol de Libro.

Bowers, C., Kreutzer, C., Cannon-Bowers, J. y Lamb, J. (2017). Team Resilience as a Second-Order Emergent State: A Theoretical Model and Research Directions. Frontiers in Psychology, 8, 1360. https://doi.org/10.3389/fpsyg.2017.01360

Carrasco, I. y Espinar, I. (2008). Terapia racional emotivo-conductual y reestructuración cognitiva. En F. J Labrador (Ed), Técnicas de Modificación de Conducta (pp. 499-515). Pirámide.

Cohen M. (2004). Complementary and Alternative Medicine. The Medical Journal of Australia, 180(11), 645-646.

Cox, R. H. (2008). Psicología del deporte. Conceptos y sus aplicaciones. Panamericana.

Dosil, J. (2008). Psicología de la actividad física y el deporte. McGraw-Hill Interamericana de España S. L.

Federación Madrileña de Deportes de Invierno (n. d.). Modalidades deportivas. https://fmdi.es/

Federación Española de Deportes de Hielo. (n. d.). Deportes. https://www.fedhielo.com/
García-Mas, A. y Gimeno F. (2008). La Teoría de Orientación de Metas y la enseñanza de la Educación Física: consideraciones prácticas. Apuntes de Psicología, 26(1), 129-142.

García-Mas, A. y Vicens, P. (2003). La psicología del equipo deportivo. Cooperación y rendimiento. Revista de Psicología del Deporte, 12(59), 79-89.

Gavino, A. (2008). Establecimiento de objetivos, planificación de actividades y administración del tiempo. En F.J Labrador (Ed) Técnicas de Modificación de Conducta (pp. 483-497). Pirámide.

Gimeno, F., Buceta, J. M., Pérez-Llanta, M. C. (2001). El cuestionario "Características

Psicológicas relacionadas con el Rendimiento Deportivo" (CPRD): características psicométricas. Analise Psicologica, $7(\mathrm{XIX})$. 93113.

Gordillo, A., Molleja, E. y Solé, J. (2011). Psicología aplicada al golf de rendimiento: una propuesta de planificación Integral. Revista Internacional de Ciencias Sociales y Humanidades, 21(2), 73-93.

Llames, R. (1994). Planificació i objectius psicològics en una escola esportiva: experiència en futbol tecnificació. Educació Física i Esports, 35, 15-20.

Marsillas, S., Rial, A., Isorna, M. y Alonso, D. (2014). Niveles de rendimiento y factores psicológicos en deportistas en formación. Reflexiones para entender la exigencia psicológica del alto rendimiento. Revista Iberoamericana de Psicología del Ejercicio y el Deporte, 9(2), 353-368.

Moreau, W. J. y Nabhan, D. (2012). Organización de un trabajo multidisciplinario en un centro olímpico de alto rendimiento en los Estados Unidos. Revista de Medicina Clínica, 23(3), 343-348.

Pares, P. (1999). El nuevo libro del mushing. Susaeta.

Pradas, F. y Reverter, J. (2011). La Tecnificación en deportes individuales versus deportes colectivos. Movimiento Humano, 1(1). 75-99.

Pérez, A. y Llames, R. (2010). Aplicaciones de los conocimientos psicológicos en la iniciación deportiva. Papeles del Psicólogo, 37(3), 269-280

Real Federación Española de Deportes de Invierno. (n. d.). Mushing. Recuperado de: https://rfedi.es/Modalidad/5

Soto, J. L. (2019). Influencia de un programa de relajación muscular progresivo sobre los niveles de ansiedad estado en gimnastas aeróbicos universitarios. Apunts: Educación física y deportes, 137(3), 142. https://www.revista-apunts.com/es/hemeroteca/?article $=1887$

Toro, R. (2014). Ellis y el Constructivismo: una perspectiva crítica sobre la Terapia Racional Emotivo Conductual. Tesis Psicológicas, 9(1), 164-177.

Tutte, V., Reche, C. y Álvarez, V. (2020). Evaluación e intervención psicológica en jugadoras de hockey sobre hierba femenino. Cuadernos de Psicología del Deporte,20(1), 62-74. https://doi. org/10.6018/cpd.406651

Villamediana, A. (2018). Trabajo Psicológico en pretemporada con el Equipo Madrileño de Mushing y el Comité de Mushing de la Federación Madrileña de Deportes de Invierno. Revista de Psicología Aplicada al Deporte y al Ejercicio Físico, 2(2), Artículo e8. https://doi.org/10.5093/rpadef2017a10 